\title{
Cost drivers of locally advanced rectal cancer treatment-An analysis of a leading healthcare insurer
}

\author{
Fabian Grass MD ${ }^{1,2}$ (1) | Amit Merchea MD ${ }^{3} \quad$ Kellie L. Mathis $\mathrm{MD}^{1}$ | \\ Nitin Mishra MBBS, MPH ${ }^{4}$ | Herbert Heien $\mathrm{MS}^{5}$ | \\ Lindsey R. Sangaralingham MPH ${ }^{5}$ | David W. Larson MD, MBA, FACS, FASCRS ${ }^{1}$
}

${ }^{1}$ Division of Colon and Rectal Surgery, Mayo Clinic, Rochester, Minnesota, USA

${ }^{2}$ Department of Visceral Surgery, Lausanne University Hospital CHUV, University of Lausanne, Switzerland

${ }^{3}$ Division of Colon and Rectal Surgery, Mayo Clinic, Jacksonville, Florida, USA

${ }^{4}$ Division of Colon and Rectal Surgery, Mayo Clinic, Phoenix, Arizona, USA

${ }^{5}$ Robert D. and Patricia E. Kern Center for the Science of Health Care Delivery, Mayo Clinic, Rochester, Minnesota, USA

\section{Correspondence}

David W. Larson, MD, MBA, FACS, FASCRS, Division of Colon and Rectal Surgery, Mayo Clinic, 200 First St SW, Rochester, MN 55905, USA.

Email: larson.david2@mayo.edu

Funding information

The Patricia Kern Center Mayo Clinic.

\begin{abstract}
Background: To evaluate the economic burden of locally advanced rectal cancer (LARC) treatment from a society perspective through analysis of health insurancederived data of commercially insured and Medicare Advantage (MA) patients.

Methods: Retrospective cost analysis of patients undergoing rectal resection within a multimodal (neoadjuvant chemoradiation + adjuvant chemotherapy) treatment strategy between January 1, 2010 and October 31, 2018, using the claims OptumLabs Data Warehouse database.
\end{abstract}

Results: In total, 1738 (935 commercial and $803 \mathrm{MA}$ ) patients were included. Overall treatment costs totaled $\$ 230,881,746$ (on average $\$ 183653 \pm 82384$ per commercially insured and $\$ 73681 \pm 32917$ per MA patient). Cost distribution according to category (commercially insured patients) was: $29.92 \%$ related to outpatient care (follow-up visits/diagnostics), radiotherapy: $21.83 \%$, index resection: $20.62 \%$, chemotherapy: $17.44 \%$, surgical inpatient: $6.32 \%$, medical inpatient: $3.28 \%$, emergency room: $0.58 \%$. Relative cost distribution of the index resection itself differed marginally between the three approaches and was $21.49 \%$ for open, $19.30 \%$ for laparoscopic, and $20.93 \%$ for robotic surgery. Relative cost distributions of neoadjuvant, adjuvant, and outpatient treatments remained unchanged, independently of the surgical approach. This representation was similar in MA patients.

Conclusion: Index-surgery related costs were outweighed by costs related to oncological and outpatient workup/follow-up treatments independently of both surgical approach and insurance type.

\section{KEYWORDS}

cost, multidisciplinary, rectal cancer treatment

\section{1 | INTRODUCTION}


financial consequences. ${ }^{1}$ Paradigm shifts in oncological treatment (chemotherapy and radiotherapy) paired with refined surgical technique including minimally invasive surgery has had a beneficial impact on both short and long-term outcomes. ${ }^{2,3}$ In Healthcare the efforts to deliver high-quality care at a lower cost may be impacted by current treatment innovation, ${ }^{4,5}$ including new technologies such as robotics, affecting both costs and outcomes. ${ }^{6}$

Currently, robot-assisted approaches are an evolving surgical tool and technique within the field of colorectal surgery. ${ }^{7-9}$ Despite multiple favorable preliminary reports with regard to surgical ease, quality, and outcomes, recent results from a landmark multicenter randomized controlled trial failed to demonstrate a substantial benefit. ${ }^{10-12}$ Furthermore, the robotic platform has been criticized for its costly surgical supplies, a prevailing argument used against the acquisition of the new technology by many institutions. ${ }^{13,14}$ The inherent increased capital and supply expense cost of robotics must be viewed within the entire cost spectrum of treating patients with this life-threatening disease.

Previous cost-related studies have been limited to institutionally derived administrative data. However, inpatient hospital costs or charges do not represent the realized cost of care from the perspective of society. Given that the value equation includes quality outcomes over cost (to society), it remains critical to evaluate new technologies and innovation from the perspective of those who pay for such innovation. ${ }^{15}$ Furthermore for LARC, the total cost of care includes a multimodal treatment pathway, which must be evaluated as well to understand the true economic burden holistically.

This analysis of a national database of both commercially insured and Medicare Advantage (MA) patients enrolled in a large national healthcare insurance organization aimed to evaluate treatmentrelated costs for LARC 6 months before, during, and 6 months after the index surgery to identify cost drivers over the entire treatment schedule.

\section{2 | METHODS}

\subsection{Data source}

This study used deidentified administrative claims data with medical benefit design and socioeconomic status information from the OptumLabs Data Warehouse, which includes medical and pharmacy claims, enrollment records for commercial and MA enrollees (https:// www.optum.com/content/dam/optum/resources/productSheets/5302_ Data_Assets_Chart_Sheet_ISPOR.pdf Accessed December 24, 2015). ${ }^{16}$ Study data were accessed using techniques adherent to the Health Insurance Portability and Accountability Act of 1996, and because this study involved analysis of pre-existing, deidentified data, the Mayo Clinic Institutional Review Board deemed it exempt from institutional review board approval.

OptumLabs Data Warehouse (OLDW) is a national claims database containing longitudinal health information on over 100 million enrollees since 1994, spanning all races and ages. It includes people from geographically diverse regions across the US and all 50 states, but with the greatest representation from the South and Midwest US. The data includes numerous data domains, including but not limited to enrollment demographic data, pharmacy claims, both facility, and professional claims.

\subsection{Patient identification}

Data on 15975 patients of at least 18 years of age having a proctectomy for rectal cancer from January 1, 2010 through October 31, 2018 were extracted. Patients were classified into open, laparoscopic, and robotic cohorts based upon CPT, ICD-9, and ICD-10 procedure and diagnosis codes. The index hospitalization of the cohort was defined as the first hospitalization for rectal resection after being diagnosed with rectal cancer. A 6-month baseline was used before the admission date and patients without rectal cancer during this time were excluded. Enrollment of 6 months before admission and 6 months post-discharge was required for all patients to be retained for further analysis. Any patient who underwent a proctectomy before their index admission were excluded. To help mitigate potential coding or missing claim errors, exclusion criteria to certain index hospitalizations were applied. Excluded were hospitalizations occurring in a skilled nursing facility, hospitalizations where the procedural technique was not easily defined, and hospitalizations resulting in negative or outlier costs. To better ensure radiation and chemotherapy costs were accounted for among patients who underwent standard treatment for LARC, patients who did not have both preoperative radiation and postoperative chemotherapy were excluded.

\subsection{Outcomes}

The distribution of the total study period cost by utilization was the primary measure of interest. From OLDW all patient claims were extracted, and total costs were found by a technique of sub-setting patients' medical claims until costs of the only outpatient and related claim types were left. In the first subset of the claims, radiation therapy and chemotherapy costs were extracted from all patient claims and set aside. The second pass set aside inpatient claims and split them up into two categories: surgical and medical. Importantly, index resection related costs (which were included in the 12-months study period) were treated as a separate entity (unrelated to inpatient medical/surgical inpatient claims). Surgical inpatient costs were based on the DRG codes for surgical procedures (ie, ileostomy takedown) and related costs, while medical inpatient costs were related to nonsurgical hospital stays and nonsurgical procedures. Thirdly, emergency room visits (defined as unplanned pre- and/or postoperative visits with/without subsequent readmission to inpatient care) were taken out, and lastly outpatient and other claims (including follow-up and diagnostics) were left. Sub-setting costs in this way ensured that costs were not overlaid or double-counted 
when chemotherapy or other visit services were intermixed. To further compare costs accurately, patients' insurance types were separated between private commercial and MA to ensure specific analysis of both fee schedules.

\section{4 | Statistical analysis}

After patient cohorts and cost measures were constructed for open, laparoscopic, and robotic surgery, each procedure technique was examined independently using pie charts to determine a distribution of costs 6 months before and after surgery. Costs were broken up into six groups: radiation, chemotherapy, surgical inpatient, medical inpatient, emergency room, and other (outpatient/follow-up/ diagnostics). Baseline and follow-up costs were summarized into separate pie charts for each resection technique to understand the cost distribution and how different they were between these two timelines. To illustrate how the baseline and follow-up cost compared to the index resection, a pie chart over the 12-month study period was considered for each resection technique.

\section{3 | RESULTS}

The flowchart of patients undergoing rectal resection for cancer is displayed in Figure 1. Nine-hundred and thirty-five unique commercially insured patients and 803 MA patients meeting all inclusion criteria were identified across all three surgery types from January 1 , 2010 to October 31, 2018. Open resection consisted of 464 patients (49.6\%), laparoscopic resection consisted of 321 patients (34.3\%) and robotic resection consisted of 150 patients (16.1\%) in the commercial group. For MA patients, the distribution was as follows: open 419 (52.2\%), laparoscopic 250 (31.1\%), robotic 134 (16.7\%). Mean length of index stay in the commercially insured group was $6.9 \pm 3.7$ days after open, $5.8 \pm 3.4$ days after laparoscopic, and $5.6 \pm 3.8$ days after robotic surgery $(p<.001)$. In MA patients, it averaged $8.4 \pm 5.2$ after open, $6.8 \pm 4.7$ after laparoscopic, and $6.5 \pm 4.2$ after robotic surgery $(p<.001)$.

Utilization-related total costs and average costs per patient are displayed in the Online Appendices for both fee schedules for all three study periods ( 6 months before admission (baseline), 6 months postdischarge, and the entire 12 months study period including index resection. Overall treatment costs totaled $\$ 230881746$, of which $\$ 49888222$ (21.61\%) were directly related to the index resection.

Commercial costs for the treatment of all 935 patients totaled $\$ 171715664$. This number was composed of a baseline total cost of $\$ 60599542$, which was mostly made up of radiation therapy (49\%) and outpatient related visit types (35\%). Follow-up total costs (\$75735948) consisted mainly of outpatient related (40\%) and chemotherapy (31\%) costs. When displaying the entire study period (12 months), the total index resection cost of \$35416134 represented 21\%, outpatient related 30\% ( $\$ 51379316$ ), radiation $22 \%$ ( $\$ 37482824)$, and chemotherapy 17\% (\$29 953889) of the total costs. Relative cost distribution overall and for the respective surgical approaches in the respective treatment period are displayed in Figure 2A-C.

For MA patients, costs for the 803 patients totaled \$59 166082 . Baseline costs were mainly due to radiation therapy (48\%) and outpatient related visit types (27\%), while post-surgery costs were mainly related to outpatient/follow-up visits and chemotherapy in the followup period ( $31 \%$ and $23 \%$, respectively). The index resection costs represented $25 \%$ when considering the entire 12 -month study period, while chemo-/radiotherapy contributed $37 \%$ (Figure 3A-C).

Relative cost distribution of the index resection itself differed marginally between the three approaches and was $21.49 \%$ for open surgery, $19.30 \%$ for laparoscopic surgery, and $20.93 \%$ for robotic surgery in the commercial group and $25.51 \%, 22.98 \%$, and $24.01 \%$ in the MA group, respectively. Similarly, relative cost distributions of neoadjuvant and adjuvant treatments remained stable independent of the surgical approach and insurance type (Online Appendices and Figures 2 and 3, respectively).

\section{4 | DISCUSSION}

Cost in healthcare is a multilayered and complex issue that lacks public transparency and understanding among many. This study attempted to evaluate the cost of LARC treatment considering different surgical approaches within a multimodal care strategy in two distinct settings: commercial insurance type and MA plan. The use of OLDW and its associated massive patient database allowed us to amass the largest comparative data set between open, laparoscopic, and robotic surgery. However, rather than a simple cost comparison related to different surgical platforms, the present analysis aimed to provide an overview of the total economic burden related to LARC treatment by correctly placing surgery-related costs into the perspective of multidimensional oncological treatments and work-up/follow-up care. While costs related to the index hospital stay were comparable among the three surgical groups in both fee schedules, the main burden for the society seems to arise from combined oncological treatments. This has to be strongly considered in the complex, highly specialized, and dynamic field of LARC surgery and may help to remove the myopic focus of costs related to surgical innovation.

Minimally invasive techniques have become standard of care in colorectal cancer surgery in high volume centers for many reasons. Most importantly, limited trauma to the abdominal wall resulted in better, sustained pain control, decreased associated wound complications, increased functional recovery, and better cosmetic results without compromising long term outcome. ${ }^{17-19}$ However, despite obvious advantages, laparoscopy has been criticized for an increased learning curve, cost of equipment acquisition and maintenance, and safety considerations. ${ }^{20,21}$ As a matter of fact, the present analysis suggests that half of Americans are still treated by an open approach. In the past surgical innovation has come under scrutiny with early series in laparoscopic colorectal surgery demonstrating the increased cost of care. ${ }^{22-24}$ Overtime favorable cost curves were 


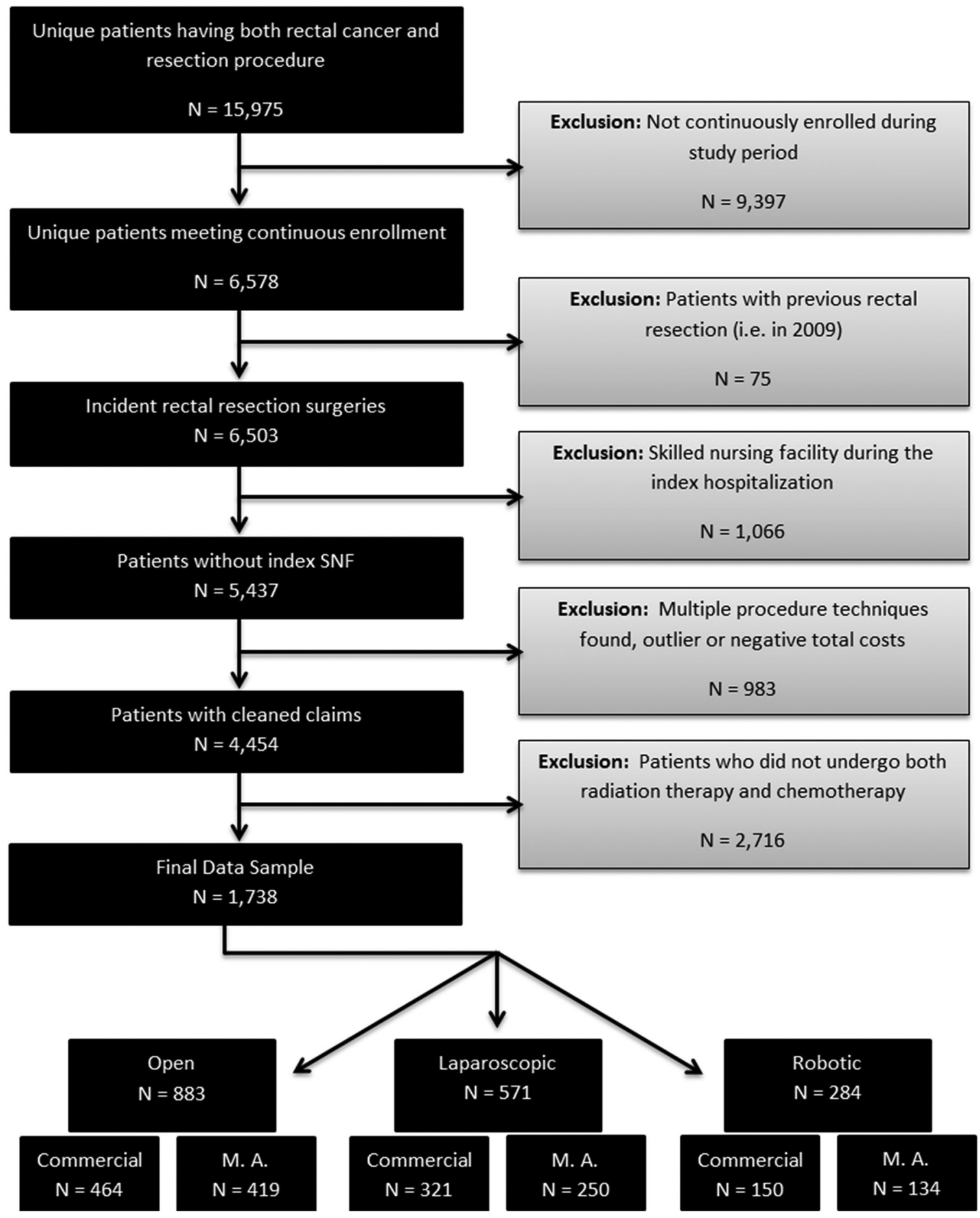

FIGURE 1 Flowchart. MA, Medicare Advantage; SNF, skilled nursing facility

identified within laparoscopy likely due to improvements in technology, education, and learning curve. ${ }^{15}$ While most surgeons in well-resourced countries would nowadays agree on the undeniable benefits of laparoscopy as compared to open surgery, the robotic platform aims to overcome the challenges of operating on LARC in a deep and narrow pelvis. ${ }^{10}$ Arguably, fixed costs related to the robotic platform including purchase and maintenance demand an initial and sustained financial effort, ${ }^{25}$ and this new technology must ultimately prove its value to patients, providers, and payers of healthcare. Taking into account intraoperative quality metrics such as conversion rate and postoperative short-term outcomes including 30-day complications and length of hospital stay, preliminary data suggest cost-efficacy with the robotic platform. ${ }^{15,26-28}$ Furthermore, a shorter learning curve compared to laparoscopy and shorter operative times with growing experience may help to decrease costs further. ${ }^{29,30}$ A singular series by Hollis et al. ${ }^{31}$ utilized a propensity 
(A) 6 months baseline

" RADIATION
= OUTPATIENT OTHER
= CHEMOTHERAPY
= SURGICAL INPATIENT
" MEDICAL INPATIENT
"EMERGENCY ROOM

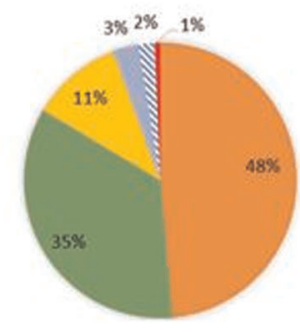

Overall

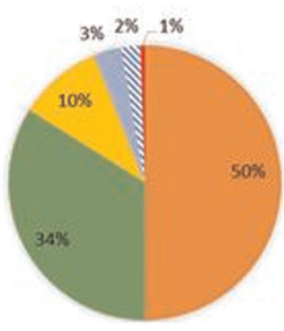

Open

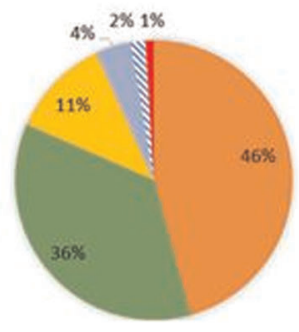

Laparoscopy

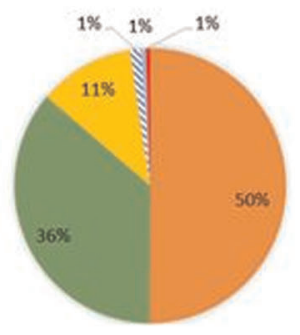

Robotic

(B) 6 months follow-up

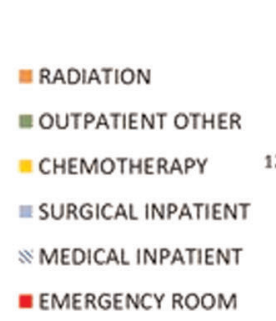

- EMERGENCY ROOM

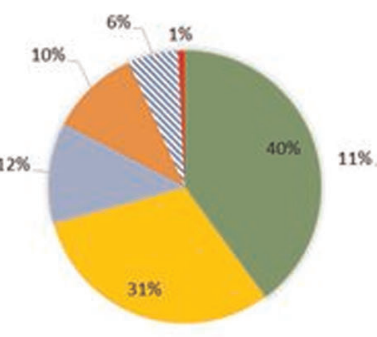

Overall

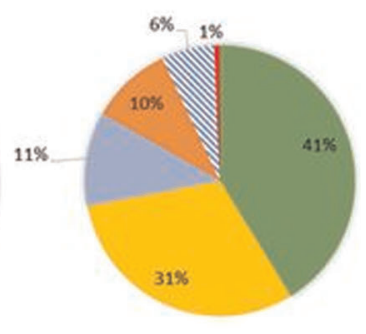

Open

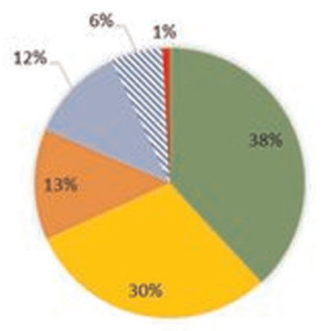

Laparoscopy

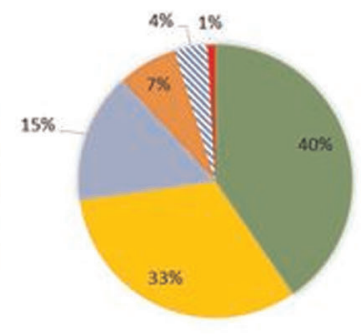

Robotic

\section{(C) 12 months study period}

" OUTPATIENT OTHER
" RADIATION
= INDEX RESECTION
" CHEMOTHERAPY
" SURGICAL INPATIENT
" MEDICAL INPATIENT
- EMERGENCY ROOM

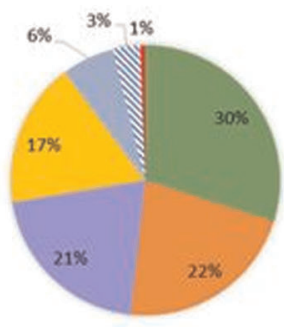

Overall

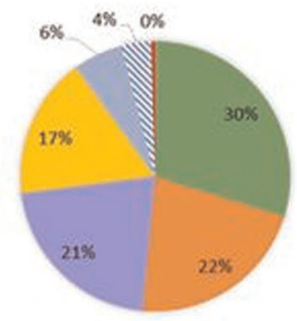

Open

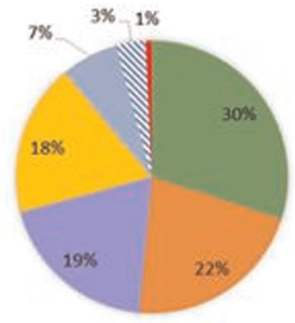

Laparoscopy

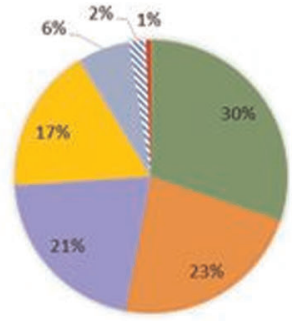

Robotic

FIGURE 2 Cost distribution of commercially insured patients. Funnel logic for claims cost extraction of commercially insured patients. Relative cost distribution during (A) the 6 months baseline period, (B) the 6 months follow-up period, and (C) the entire 12 months study period (including index resection-related costs) overall and for the three different surgical approaches. Displayed are cost contributions in percent [Color figure can be viewed at wileyonlinelibrary.com]

score to match laparoscopic and robotic colorectal cases with open surgery. They demonstrated median hospital costs were similar between laparoscopic and open and between robotic and open surgery, which is similar to our series revealing similar cost distribution associated with all three surgical platforms.

This series reviewed patients for their entire episode of rectal cancer care and evaluated the total cost of care from a societal perspective, including oncological treatments related to the multifactorial treatment of rectal cancer. Furthermore, this analysis specifically considered both health insurance plans, taking into account the unique setting of MA covering patients with more complex medical needs. Unlike previous work evaluating administrative costs related to robotic surgery, this paper uniquely evaluated the cost to society and payors of healthcare rather than isolating the costs to institutions. Interestingly, costs of radiotherapy and chemotherapy outweighed direct index surgery stay related costs in all surgical settings. Costs related to oncological treatments and work-up/ follow-up must be considered as real cost drivers over the long-term within the overall value equation. In other words, from the social standpoint, we must find ways to lower the costs of care, but in particular, that of chemotherapy and radiation as well as outpatient follow up in order that greater value is achieved to society at large.

This series has several limitations, including the challenges and biases of any retrospective database review. The present study analyzed costs associated with both treatment cost and insurance reimbursement cost. Since MA does not reimburse as much as commercial insurance types, both fee schedules were separated out and compared on the same scale. This is reflected by the generally lower overall costs for MA patients in this study. The possibility of additional confounding variables that cannot be elucidated through this data warehouse approach cannot be excluded. Moreover, utilizing this data warehouse, no specific clinical variables which may 
(A) 6 months baseline

$=$ RADIATION
$=$ OUTPATIENT OTHER
$=$ CHEMOTHERAPY
$=$ SURGICAL INPATIENT
"MEDICAL INPATIENT
$=$ EMERGENCY ROOM

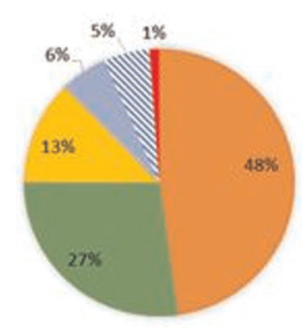

Overall

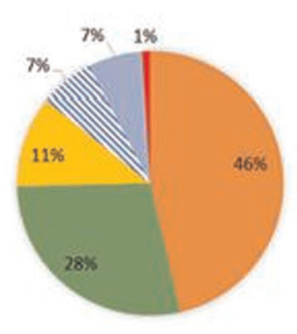

Open

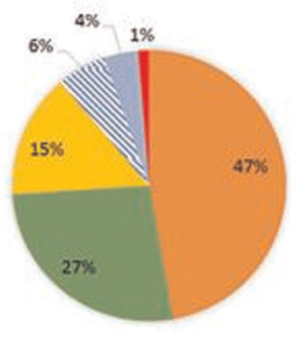

Laparoscopy

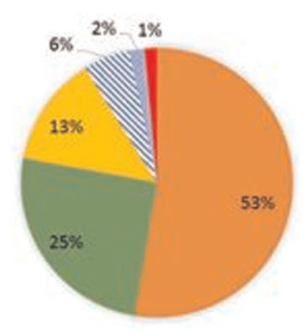

Robotic

(B) 6 months follow-up

\section{RADIATION = OUTPATIENT OTHER = CHEMOTHERAPY = SURGICAL INPATIENT «MEDICAL INPATIENT - EMERGENCY ROOM}

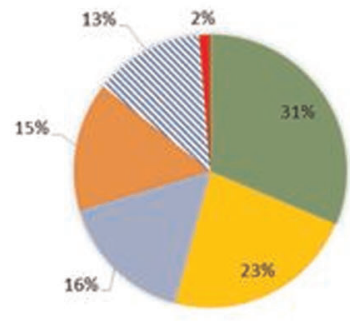

Overall

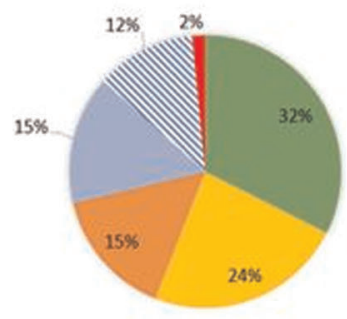

Open

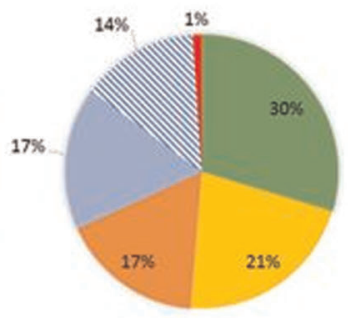

Laparoscopy

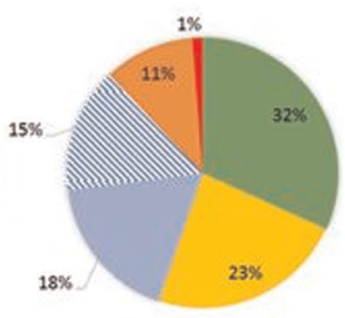

Robotic

\section{(C) 12 months study period}

$=$ OUTPATIENT OTHER
$=$ RADIATION
$=$ INDEX RESECTION
$=$ CHEMOTHERAPY
$=$ SURGICAL INPATIENT
\& MEDICAL INPATIENT
= EMERGENCY ROOM

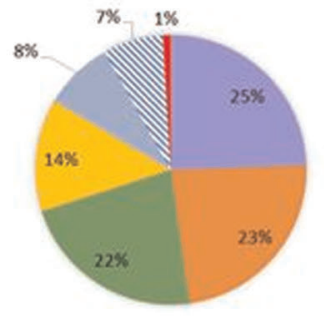

Overall

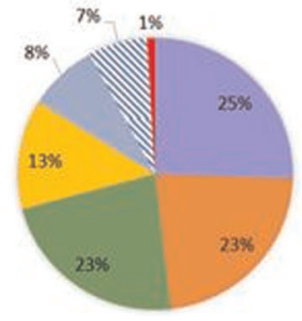

Open

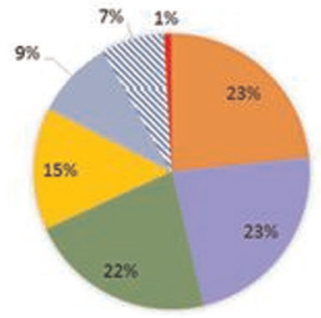

Laparoscopy

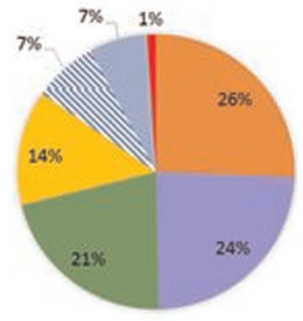

Robotic

FIGURE 3 Cost distribution of MA patients. Funnel logic for claims cost extraction of MA patients. Relative cost distribution during (A) the 6 months baseline period, (B) the 6 months follow-up period, and (C) the entire 12 months study period (including index resection-related costs) overall and for the three different surgical approaches. Displayed are cost contributions in percent. Medicare Advantage [Color figure can be viewed at wileyonlinelibrary.com]

have significantly impacted the cost of care including associated complications of any surgical approach were detailed. Furthermore, surgical interventions based on individual surgeon characteristics or institutional characteristics of care were not evaluated. It is quite possible that advancing technical operations are more likely to be performed at high-volume practices which may, as indicated by previous literature, have improved outcomes. ${ }^{32}$ Lastly, further discriminating between clinical follow-up visits and more expensive diagnostics (ie, magnetic resonance imaging), which were regrouped as outpatient costs for the purpose of this study, was not possible. However, evaluating the cost burden of diagnostic procedures may be possible in future analyses through algorithms based on timing.

In conclusion, this series demonstrates similar relative cost distribution among open, laparoscopic, and robotic LARC surgery embedded in a multimodal pathway, in both commercial and Medicare insurance fee schedules. Cost drivers from a society perspective seem not to be related to surgical innovation such as the robotic platform, but rather arise from oncological treatments and expensive work-up and follow-up management and diagnostic procedures. This information places into proper context the growth and expansion of innovative surgery such as robotics. These findings should limit our misplaced perception that innovation is leading to the increased cost of care and rather focus on reducing total costs of care by providing ever-increasing quality and limiting unnecessary intervention and follow-up.

\section{ACKNOWLEDGMENT}

This work was supported by the Patricia Kern Center Mayo Clinic.

\section{CONFLICT OF INTERESTS}

The authors declare that there are no conflict of interests. 


\section{DATA AVAILABILITY STATEMENT}

The administrative claims data utilized for this analysis derived from the OptumLabs Data Warehouse and will not be shared.

\section{ORCID}

Fabian Grass (D) https://orcid.org/0000-0001-9884-2879

\section{REFERENCES}

1. Salem ME, Hartley M, Unger K, Marshall JL. Neoadjuvant combinedmodality therapy for locally advanced rectal cancer and its future direction. Oncology. 2016;30(6):546-562.

2. Sineshaw HM, Jemal A, Thomas CR, Jr., Mitin T. Changes in treatment patterns for patients with locally advanced rectal cancer in the United States over the past decade: an analysis from the National Cancer Data Base. Cancer. 2016;122(13):1996-2003.

3. Jeong SY, Park JW, Nam BH, et al. Open versus laparoscopic surgery for mid-rectal or low-rectal cancer after neoadjuvant chemoradiotherapy (COREAN trial): survival outcomes of an open-label, non-inferiority, randomised controlled trial. Lancet Oncol. 2014;15(7):767-774.

4. Lievens $\mathrm{Y}$, Audisio R, Banks I, et al. Towards an evidence-informed value scale for surgical and radiation oncology: a multi-stakeholder perspective. Lancet Oncol. 2019;20(2):e112-e123.

5. Mahvi DA, Liu R, Grinstaff MW, Colson YL, Raut CP. Local cancer recurrence: the realities, challenges, and opportunities for new therapies. CA Cancer J Clin. 2018;68(6):488-505.

6. Brazier JE, Johnson AG. Economics of surgery. Lancet. 2001; 358(9287):1077-1081.

7. Rausa E, Bianco F, Kelly ME, et al. Systemic review and network meta-analysis comparing minimal surgical techniques for rectal cancer: quality of total mesorectum excision, pathological, surgical, and oncological outcomes. J Surg Oncol. 2019;119(7):987-998.

8. Simillis C, Lal N, Thoukididou SN, et al. Open versus laparoscopic versus robotic versus transanal mesorectal excision for rectal cancer: a systematic review and network meta-analysis. Ann Surg. 2019;270:59-68.

9. Sheetz KH, Norton EC, Dimick JB, Regenbogen SE. Perioperative outcomes and trends in the use of robotic colectomy for medicare beneficiaries from 2010 through 2016. JAMA Surg. 2019;155:41-49.

10. Baek JH, McKenzie S, Garcia-Aguilar J, Pigazzi A. Oncologic outcomes of robotic-assisted total mesorectal excision for the treatment of rectal cancer. Ann Surg. 2010;251(5):882-886.

11. Lightner AL, Kelley SR, Larson DW. Robotic platform for an IPAA. Dis Colon Rectum. 2018;61(7):869-874.

12. Jayne D, Pigazzi A, Marshall $H$, et al. Effect of Robotic-assisted vs conventional laparoscopic surgery on risk of conversion to open laparotomy among patients undergoing resection for rectal cancer: the ROLARR randomized clinical trial. JAMA. 2017;318(16):1569-1580.

13. Zelhart M, Kaiser AM. Robotic versus laparoscopic versus open colorectal surgery: towards defining criteria to the right choice. Surg Endosc. 2018;32(1):24-38.

14. Sheetz KH, Dimick JB. Is it time for safeguards in the adoption of robotic surgery? JAMA. 2019;321(20):1971-1972.

15. Cleary RK, Mullard AJ, Ferraro J, Regenbogen SE. The cost of conversion in robotic and laparoscopic colorectal surgery. Surg Endosc. 2018;32(3):1515-1524.

16. Wallace PJ, Shah ND, Dennen T, Bleicher PA, Crown WH. Optum Labs: building a novel node in the learning health care system. Health Aff. 2014;33(7):1187-1194.

17. Bonjer HJ, Deijen CL, Abis GA, et al. A randomized trial of laparoscopic versus open surgery for rectal cancer. N Engl J Med. 2015; 372(14):1324-1332.
18. van der Pas $\mathrm{MH}$, Haglind $\mathrm{E}$, Cuesta MA, et al. Laparoscopic versus open surgery for rectal cancer (COLOR II): short-term outcomes of a randomised, phase 3 trial. Lancet Oncol. 2013;14(3):210-218.

19. Goh BK. Impact of laparoscopic resection for colorectal cancer on operative outcomes and survival. Ann Surg. 2007;246(2):338-339.

20. Fleshman J, Branda M, Sargent DJ, et al. Effect of laparoscopicassisted resection vs open resection of stage II or III rectal cancer on pathologic outcomes: The ACOSOG Z6051 randomized clinical trial. JAMA. 2015;314(13):1346-1355.

21. Stevenson ARL, Solomon MJ, Lumley JW, et al. Effect of laparoscopic-assisted resection vs open resection on pathological outcomes in rectal cancer: the ALaCaRT randomized clinical trial. JAMA. 2015;314(13):1356-1363.

22. Pfeifer J, Wexner SD, Reissman P, et al. Laparoscopic vs open colon surgery. Costs and outcome. Surg Endosc. 1995;9(12):1322-1326.

23. Leung KL, Kwok SP, Lam SC, et al. Laparoscopic resection of rectosigmoid carcinoma: prospective randomised trial. Lancet. 2004; 363(9416):1187-1192.

24. Dowson HM, Gage H, Jackson D, Qiao Y, Williams P, Rockall TA. Laparoscopic and open colorectal surgery: a prospective cost analysis. Colorectal Dis. 2012;14(11):1424-1430.

25. Tandogdu Z, Vale L, Fraser C, Ramsay C. A systematic review of economic evaluations of the use of robotic assisted laparoscopy in surgery compared with open or laparoscopic surgery. Appl Health Econ Health Policy. 2015;13(5):457-467.

26. Cheng $\mathrm{CL}$, Rezac $\mathrm{C}$. The role of robotics in colorectal surgery. BMJ. 2018;360:j5304.

27. lelpo B, Duran H, Diaz E, et al. Robotic versus laparoscopic surgery for rectal cancer: a comparative study of clinical outcomes and costs. Int J Colorectal Dis. 2017;32(10):1423-1429.

28. Tam MS, Kaoutzanis C, Mullard AJ, et al. A population-based study comparing laparoscopic and robotic outcomes in colorectal surgery. Surg Endosc. 2016;30(2):455-463.

29. Morelli L, Guadagni S, Lorenzoni V, et al. Robot-assisted versus laparoscopic rectal resection for cancer in a single surgeon's experience: a cost analysis covering the initial 50 robotic cases with the da Vinci Si. Int J Colorectal Dis. 2016;31(9):1639-1648.

30. Byrn JC, Hrabe JE, Charlton ME. An initial experience with 85 consecutive robotic-assisted rectal dissections: improved operating times and lower costs with experience. Surg Endosc. 2014;28(11): 3101-3107.

31. Hollis RH, Cannon JA, Singletary BA, Korb ML, Hawn MT, Heslin MJ. Understanding the value of both laparoscopic and robotic approaches compared to the open approach in colorectal surgery. J Laparoendosc Adv Surg Tech A. 2016;26(11):850-856.

32. Balentine $C J$, Naik $A D$, Robinson $C N$, et al. Association of highvolume hospitals with greater likelihood of discharge to home following colorectal surgery. JAMA Surg. 2014;149(3):244-251.

\section{SUPPORTING INFORMATION}

Additional Supporting Information may be found online in the supporting information tab for this article.

How to cite this article: Grass F, Merchea A, Mathis KL, et al. Cost drivers of locally advanced rectal cancer treatment-An analysis of a leading healthcare insurer. J Surg Oncol. 2021;123: 1023-1029. https://doi.org/10.1002/jso.26390 